\title{
Improved Edge Detection based Fast Face Detection Method using Enhanced Fourier Transform (IED-FFD) towards Facial Expression Recongnition
}

\author{
DINESH KUNAR P \\ Ph.D. Research Scholar, PG and Research Department of Computer Science \\ Dr. N.G.P. Arts and Science College, Coimbatore \\ Dr. B. Rosiline Jeetha \\ Professor and Head, PG and Research Department of Computer Science \\ Dr. N.G.P. Arts and Science College, Coimbatore
}

\begin{abstract}
Facial expression recognition extends in various real world scenario that includes safety, scientific and many commercial applications. Face detection through edge detection is focussed in this research before recognizing facial expressions. Hence, in this paper, an improved sobel operator is employed. The improved sobel operator makes use of geodesic distance. To identify the position of edge, the minimum distance between the image and the elements of features with the help of geodesic distance are used. After that, an enhanced fast fourier transform (E-FFT) is employed for face detection. Performance metrics such as time taken for edge detection, time taken for face detection and accuracy are taken into account. From the results it is evident that the proposed work consumes less time for edge detection and face detection. Also it is noteworthy that the accuracy is improved significantly.
\end{abstract}

Keywords: Facial expression recognition, edge detection, face detection, fast fourier transform, accuracy.

\section{INTRODUCTION}

Face is a vital part is social communications. Ekman and Frisen [18] mentioned that, happy, sad, anger, fear, disgust and surprise are the six basic expressions that are freely documented across diverse cultures. A system developed for contemplating facial reactions mechanically through a human-computer interaction, is known as Facial expression recognition. Edges are basically the noticeable variation of intensities in an image. Edges help to identify the location of an object and the boundary of a particular entity in the image. It also helps in feature extraction and pattern recognition. Hence, edge detection is of great importance in computer vision. So far, most of the researchers have chosen software for implementation of basic edge detection algorithms and their variations [19]. But, it has been established that it is not an efficient approach for real time applications. Face detection helps in FER method for faster recognition of human expressions. This research article proposes Improved Edge Detection based Fast Face Detection Method using Enhanced Fourier Transform (IED-FFD) towards Facial Expression Recongnition. This paper is organized as follows. Section 2 gives a brief review of literature. Section 3 describes the proposed work. Section 4 provides results and discussions. Section 5 offers concluding remarks.

\section{RELATED WORKS}

Amer et al. [1] compared all the edge detection algorithms to study their performances. The results showed that Sobel and Canny edge detectors are not as much of sensitive to random noise in an image as compared to Robert's and Prewitt operators. Cui et al. [2] deliberated the recompenses and difficulties of edge detection algorithms and matched them. The evaluationindicates that binary morphology operator attains better results. A system namely bordering close has also been used so as to achieve clear and integral image profile. [3] Deng et al. developed an algorithm in which fusion technology is used. In this an improved Sobel operator is introduced which is combined with wavelet transform, canny and prewitt operators. The algorithm effectively improved the results of edge detection. Chinu et al.[4] discuss a hybrid strategy for color based image edge detection. This approach has been mainly implemented for overcoming poor edge localization, high sensitivity to noise, edge detection of images with complex background and detecting color edges. Both sequential and parallel approaches have been used and compared which depicted that parallel strategy shows a performance gain of $68 \%$. To measure the accuracy of edge detection various metrics are used like PSNR, speed up, efficiency etc. [5]. An algorithm is developed in which improved canny operator is used. In this improved operator, morphological filter replaces the Gaussian filter. The morphological filtering pre-treats the noise of image, keeps the edge strength and details, thus achieving better accuracy. Gao et al.[6] proposed a method in which Sobel edge detection is combined with soft-threshold wavelet de-noising on images which have White Gaussian 
noises. Firstly, soft-threshold wavelet denoising is used to remove the noises from the image and then Sobel operator is applied to it. The results show an improvement in the accuracy of edge detection.

As far as related works in face detection is concerned, recently, many appearance based face recognition systems are developed. Such systems aimsto lessen the computation time that is required for detection of face images [13]. The appearance based face recognition systems use face image pixel intensity values directly as the feature values for recognition and are represented using single valued variable. But such single valued variables may not be able to capture the variation of feature values of the images of the same subject and will also have high dimensional features data. One of the techniques adapted by researchers to develop efficient system with reduced number features is method- ology of feature learning. Feature learning or active learning plays a major role to discriminately represent or construct training image data (extracted feature) to improve the performance of classification task [7-10]. Hence, recently a number of active learning methods have been proposed which combine representiveness and informativeness of training samples to attain better classification accuracy [8]. Chong Peng et al. propose a supervised feature learning model for classification of both low and high dimensional data [11]. The technique estimates regression vector using a dis- criminative regression approach to estimate the similarity between the test data and training data. However, classification task be- comes challenging, when low or high dimensional image data are affected by distortions such as illumination, pose variation, presence of occlusion and geometrical variations etc. [12]. To handle such a class of distortions, a mathematical framework for multi- class classification of high dimensional image data has been pro- posed. The minimax framework estimates an optimal representation model that minimizes the fitting error under listed distortions to the data in an application of interest. Further, categorical information is derived based on the estimated model and regression models used for classification [12].

During the contemporary years several research works are carried out as far as face recognition is concerned. Many researchers aim to reduce the time complexity in terms of computation time taken for facial image detection. It is to be noted that there are face recognition systems that make use of face image pixel intensity values directly as the feature values for recognition. Such systems / methods the representation made use of single valued variable. In some literature, it is mentioned that single valued variables are not capable enough to seizure the variation of feature values of the facial images that has high dimensional features.

\section{PROPOSED WORK}

\subsection{Improved Sobel Operator for Edge Detection in Facial Expression Recognition Images}

The sobel operator is a famous edge detection mechanism and can be applied to wide variety of images that includes digital color images and grayscale images. In this section, a brief introduction about sobel operator for edge detection is presented and then the improvement work is presented later. The sobel operator has a couple of $3 \times 3$ convolution kernels and is depicted in Fig.1.

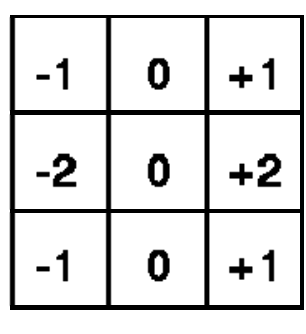

Gx

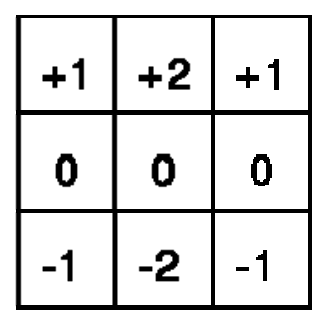

Gy

Fig.1. Convolution kernel in Sobel operator

The Sobel operator's kernel structure helps to retort in the best way to edges running vertically and horizontally based on the pixel grid, one kernel for each of the two perpendicular orientations. These kernels are applied distinctly to the input facial expression image in order to obtain discrete measurements of the incline component in each orientation and represented as $G_{x}$ and $G_{y}$. In this work, these are embeeded together for discovering the complete magnitude of the incline at each point and the orientation of that incline. The incline magnitude is mathematically represented as

$|G|=\sqrt{G x^{2}+G y^{2}} \ldots$

Inorder to quickly process, the equation (1) is modified as

$|G|=\left|G_{x}\right|+\left|G_{y}\right| \cdots$ 
The angle of direction of the edge for spatial incline is represented as,

$\theta=\arctan \left(\frac{G_{y}}{G_{x}}\right) \ldots$

It is considered that $\mathrm{g}_{\mathrm{i}} \mathrm{is}$ the $\mathrm{i}^{\text {th }}$ image where $i \in[0,1]$. In order to identify the position of edge, the minimum distance between the image and the elements of features are used and is given as,

$d_{\text {pos }}^{*}=\arg \min p\left(d_{\text {pos }} \mid \operatorname{dist}\left(F_{n}, \varphi_{\text {img }}\right) \ldots\right.$

In this work, the geodesic distance is used. In the input facial expression image $I(x)$, the geodesic distance $D\left(x_{i}, x_{j}\right)$ between the pixels $x_{i}$ and $x_{j}$ is defined as the shortest path that connects $x_{i}$ and $x_{j}$. It is mathematically represented as,

$$
D\left(x_{i}, x_{j}\right)=\min _{\Gamma \in P_{x_{i}, x_{j}}} \int_{0}^{l(\Gamma)} \sqrt{1+\gamma^{2}\left(\nabla I(s) \cdot \Gamma^{\prime}(s)\right)^{2} d s} \ldots
$$

In the equation (5), $P_{x_{i}, x_{j}}$ is the set of all available paths between $x_{i}, x_{j}, \Gamma$ is the parameterized depiction by arclength on the path, $\nabla I(s)$ is the incline value, $\Gamma^{\prime}(s) \quad$ is the unit vector tangent to the direction of the path. The factor $\gamma$ weights the influence of the image incline versus spatial distance. The input facial expression image is the main processing target, and the geodesic distance is computed only by accumulating neighboring pixel values as in equation.6.

$$
d(\Gamma)=\sum_{i=1}^{n} d_{i, i-1}=\sum_{i=1}^{n} I\left(p_{i}\right)-I\left(p_{i-1}\right) \ldots
$$

\subsection{Face Detection using Enhanced Fast Fourier Transform for Facial Expression Recognition}

In this section the face detection for facial expression recognition is presented. Once after the edges are detected in the given facial image, local variations in spatial domain is extracted by making use of frequency domain transform. The unalike brightness, expression dissimilarities and constrictions of face images are denoted in frequency domain by making use of Enhanced Fast Fourier Transform (E-FFT) that produces the features. The E-FFT is represented as,

$$
\Gamma(k, l)=\sum_{m=0}^{M-1} \sum_{n=0}^{N-1} \tau(m, n) e^{-j \frac{2 \pi}{M} m k-\frac{2 \pi}{N} n l} \ldots
$$

In the equation (7), $\Gamma(k, l)$ denotes the image co-efficient; $(m, n)$ denotes the position of RGB value of image $\Gamma$.Additionally, low frequency components are saved for excerpting discerning features that are required for face detection. The maximum magnitude value is taken as the feature descriptor of the facial image. This research work makes use of altered feature representation when comapred with other facee recognition systems as described in the related work. This work also incorporates a single feature value to represent the entire face image. Moreover, symbolic items which fit to the same course are denoted by a interval valued variable i.e. by maximum and minimum magnitude values of $\mathrm{N}$ symbolic course of the corresponding face class. For the symbolic similarity analysis between trained symbolic objects and test object, a new symbolic similarity measure is devised and employed in this work. 


\section{Results and Discussions}

Table - 1: Overall Performance Analysis of the proposed IED-FFD with existing works

\begin{tabular}{|c|c|c|c|c|c|c|c|}
\hline \multirow{2}{*}{ Image Title } & \multicolumn{2}{|c|}{$\begin{array}{l}\text { Elapsed Time for Edge } \\
\text { Detection (in seconds) }\end{array}$} & \multicolumn{2}{|c|}{$\begin{array}{l}\text { Elapsed Time for Face } \\
\text { Detection (in seconds) }\end{array}$} & \multirow{2}{*}{$\begin{array}{l}\text { Image } \\
\text { Title }\end{array}$} & \multicolumn{2}{|c|}{ Accuracy (in \%) } \\
\hline & GETF [14] & IED-FFD & $\begin{array}{c}\text { LS-KC- } \\
\text { SVM [15] }\end{array}$ & IED-FFD & & $\begin{array}{l}\text { LS-KC- } \\
\text { SVM [15] }\end{array}$ & IED-FFD \\
\hline Image 01 & 0.563 & 0.279 & 0.640 & 0.405 & Image 01 & 81.63 & 89.88 \\
\hline Image 02 & 0.562 & 0.284 & 0.678 & 0.394 & Image 02 & 83.27 & 90.70 \\
\hline Image 03 & 0.538 & 0.238 & 0.649 & 0.398 & Image 03 & 81.00 & 90.45 \\
\hline Image 04 & 0.556 & 0.285 & 0.662 & 0.423 & Image 04 & 81.99 & 90.00 \\
\hline Image 05 & 0.522 & 0.268 & 0.640 & 0.413 & Image 05 & 83.00 & 90.78 \\
\hline Image 06 & 0.559 & 0.236 & 0.666 & 0.405 & Image 06 & 82.89 & 90.50 \\
\hline Image 07 & 0.512 & 0.247 & 0.646 & 0.438 & Image 07 & 83.55 & 90.31 \\
\hline Image 08 & 0.529 & 0.263 & 0.669 & 0.421 & Image 08 & 83.06 & 89.81 \\
\hline Image 09 & 0.513 & 0.288 & 0.671 & 0.419 & Image 09 & 83.63 & 90.90 \\
\hline Image 10 & 0.517 & 0.288 & 0.675 & 0.444 & Image 10 & 81.21 & 90.22 \\
\hline Image 11 & 0.568 & 0.240 & 0.657 & 0.400 & Image 11 & 82.68 & 91.76 \\
\hline Image 12 & 0.559 & 0.288 & 0.635 & 0.433 & Image 12 & 82.99 & 89.13 \\
\hline Image 13 & 0.532 & 0.287 & 0.644 & 0.433 & Image 13 & 83.18 & 90.45 \\
\hline Image 14 & 0.577 & 0.259 & 0.685 & 0.407 & Image 14 & 81.60 & 89.79 \\
\hline Image 15 & 0.512 & 0.278 & 0.639 & 0.420 & Image 15 & 82.63 & 90.24 \\
\hline Image 16 & 0.541 & 0.239 & 0.680 & 0.385 & Image 16 & 81.70 & 89.84 \\
\hline Image 17 & 0.537 & 0.255 & 0.662 & 0.384 & Image 17 & 81.69 & 89.38 \\
\hline Image 18 & 0.564 & 0.285 & 0.690 & 0.417 & Image 18 & 81.65 & 91.33 \\
\hline Image 19 & 0.566 & 0.278 & 0.635 & 0.435 & Image 19 & 83.65 & 89.64 \\
\hline Image 20 & 0.523 & 0.288 & 0.657 & 0.445 & Image 20 & 82.96 & 89.34 \\
\hline Image 21 & 0.544 & 0.269 & 0.636 & 0.389 & Image 21 & 81.92 & 91.06 \\
\hline Image 22 & 0.541 & 0.232 & 0.688 & 0.420 & Image 22 & 83.80 & 89.46 \\
\hline Image 23 & 0.555 & 0.281 & 0.630 & 0.413 & Image 23 & 81.64 & 91.09 \\
\hline Image 24 & 0.560 & 0.286 & 0.677 & 0.381 & Image 24 & 81.94 & 91.52 \\
\hline Image 25 & 0.563 & 0.271 & 0.679 & 0.404 & Image 25 & 82.08 & 90.22 \\
\hline $\begin{array}{c}\text { Average } \\
\text { Time Taken } \\
\text { (in seconds) }\end{array}$ & 0.544 & 0.268 & 0.660 & 0.413 & $\begin{array}{l}\text { Average } \\
\text { Accuracy }\end{array}$ & 82.45 & 90.31 \\
\hline
\end{tabular}

The dataset are obtained from the openly available dataset [16]. 25 images are taken for testing the effectiveness of the proposed IED-FFD. Performance metrics such as time taken for edge detection, time taken for face detection and accuracy are taken for comparing the proposed IED-FFD method. In order to compare the time taken for edge detection, GETF [14] method is chosen for comparison. LS-KC-SVM method is chosen for comparing the effectiveness of the proposed IED-FFD in terms of time taken for face detection and accuracy.

Table - 1 presents the overall performance analysis of the existing works and proposed work. From the presented results it is evident that the proposed work IED-FFD consumes less time for edge detection when compared with GETF [14] method. The average time taken for edge detection of IED-FFD is 0.268 when compared with GETF [14] method which consumes 0.544 seconds. The results are presented in Fig. 2.

As far as the average time taken for face detection is concerned, the existing LS-KC-SVM [15] had taken 0.660 seconds whereas the proposed IED-FFD consumes 0.413 seconds. The graphical results are presented in Fig. 3. It is noteworthy that the average accuracy of IED-FFD is $90.31 \%$ which is better when compared with LS-KCSVM [15] method that obtains $82.45 \%$. The results are presented in Fig. 4. 


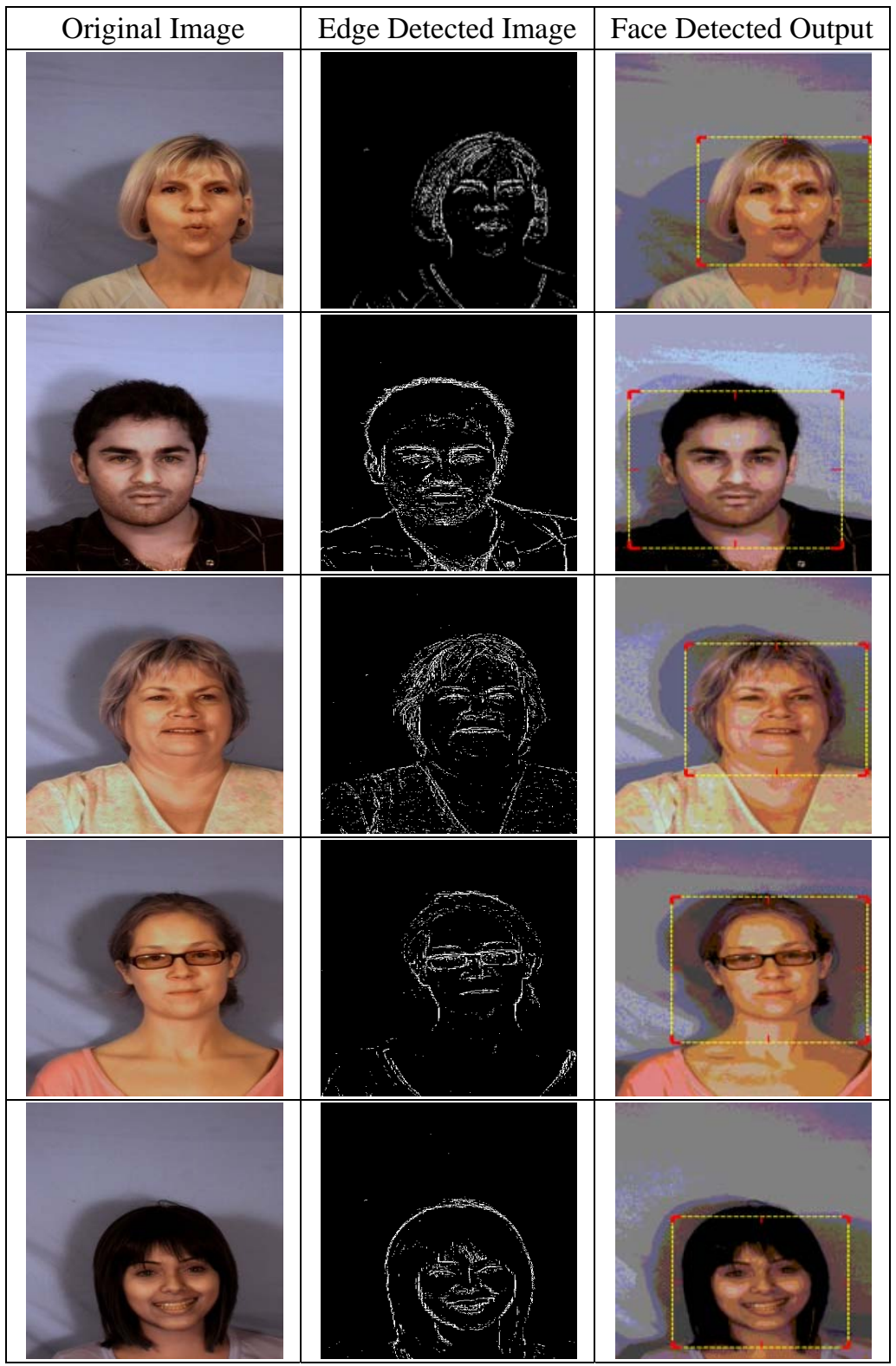

Fig.1. (a) Original Image (b) Edge Detected Image (c) Face Detected Output

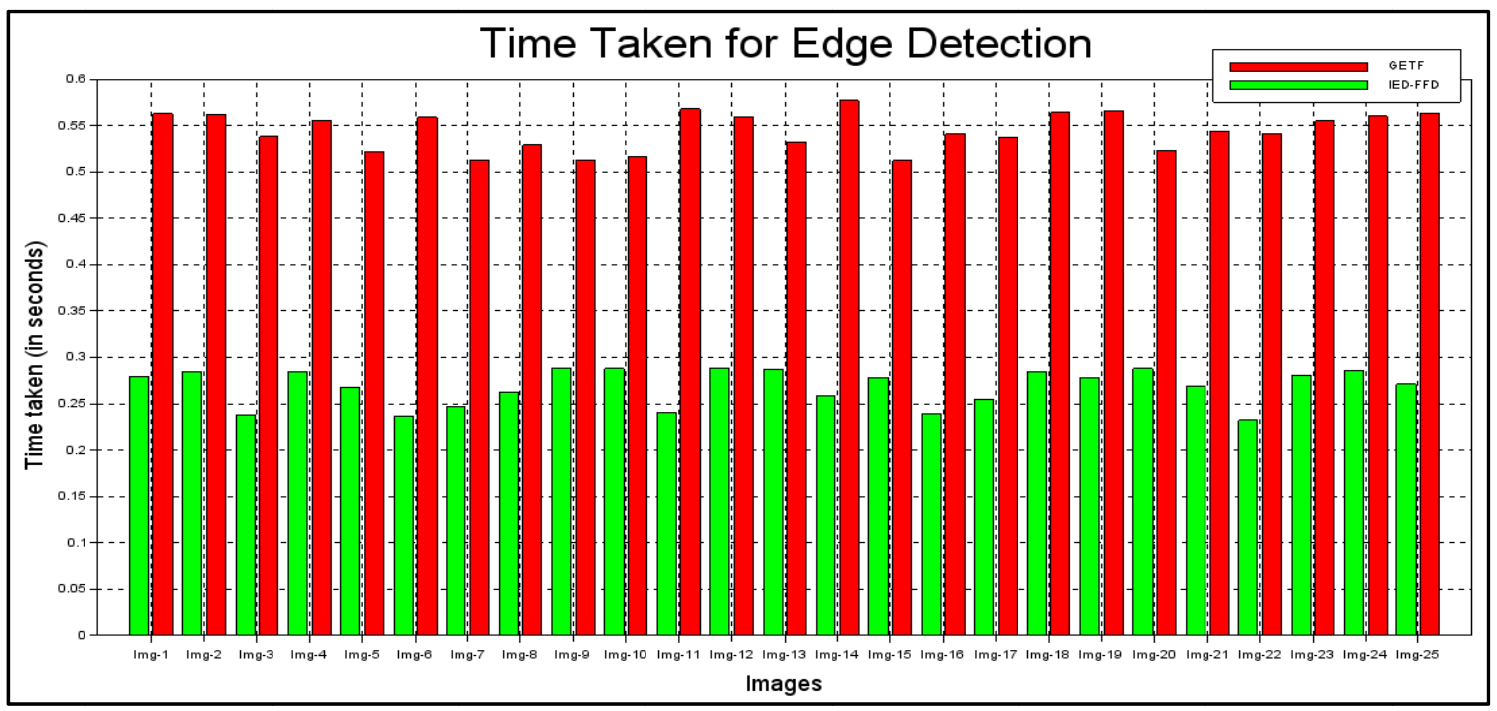

Fig. 2. Performane Analysis: Time Taken for Edge Detection 


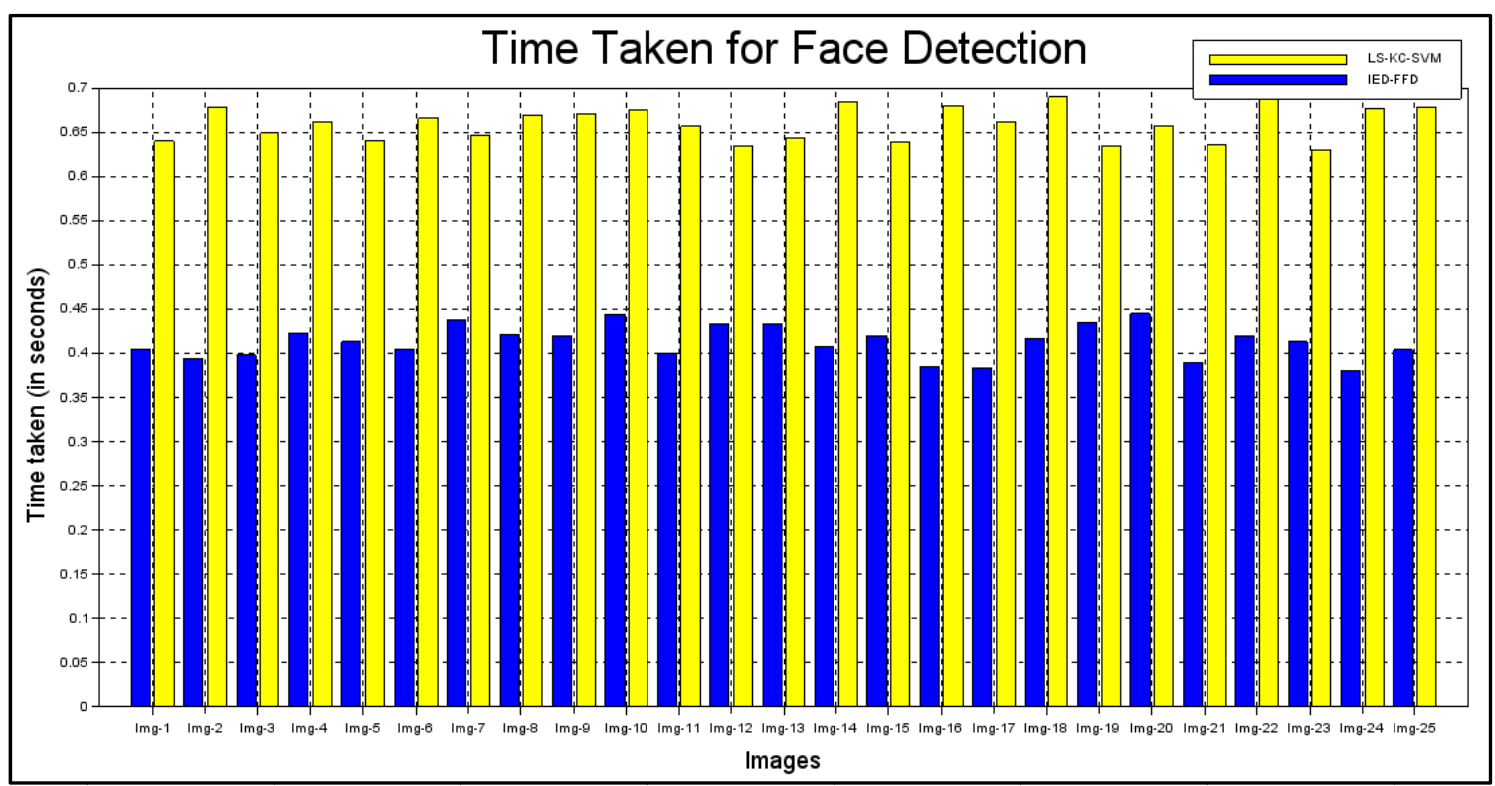

Fig. 3. Performane Analysis: Time Taken for Face Detection

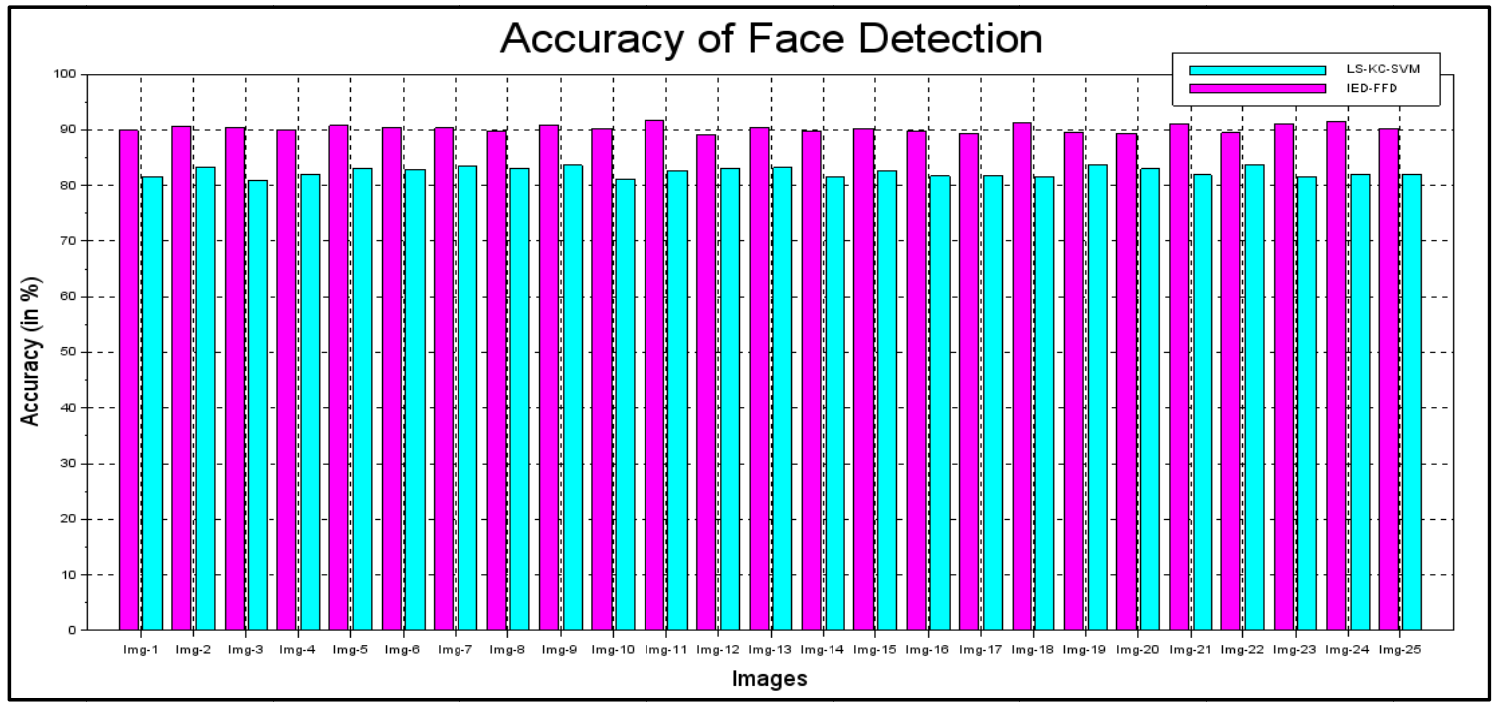

Fig. 4. Performane Analysis: Accuracy

\section{Conclusion}

Facial expression recognition system can be developed through schematic process. Usually the images obtained possibly will have noises in it. Hence the previous work focusses on unaffected serial prophecy based filter technique for noise removal in facial expression recognition images. In this second phase of research improved edge detection based fast face detection method using enhanced fourier transform (IED-FFD) towards facial expression recongnition is proposed. 25 images are taken for evaluating the performance. IED-FFD has two folds. First, an improved edge detection mechanism is used that consumes less time. Next, fast face detection is performed with improved accuracy upto $91.76 \%$ with less time.

\section{References}

[1] G. M. H. Amer, A. M. Abushaala, "Edge detection methods," $2^{\text {nd }}$ World Symposium on Web Applications and Networking (WSWAN), pp. 1-7, 2015.

[2] F. Y. Cui, L. Zou, B. Song, "Edge feature extraction based on digital image processing techniques," IEEE International Conference on Automation and Logistics, 2008, pp. 2320-2324, 2008.

[3] C. Deng, W. Ma, Y. Yin, "An edge detection approach of image fusion based on improved Sobel operator," $4^{\text {th }}$ International Congress on Image and Signal Processing, pp. 1189-1193, 2011.

[4] Chinu, A. Chhabra, "A hybrid approach for color based image edge detection," International Conference on Advances in Computing, Communications and Informatics (ICACCI), pp. 2443-2448, 2014.

[5] C. X. Deng, G. B. Wang, X. R. Yang, "Image edge detection algorithm based on improved Canny operator," International Conference on Wavelet Analysis and Pattern Recognition, pp. 168-172, 2013.

[6] W. Gao, X. Zhang, L. Yang, H. Liu, "An improved Sobel edge detection," $3^{\text {rd }}$ International Conference on Computer Science and Information Technology, pp. 67-71, 2010. 
[7] B. Du, W. Xiong, J. Wu, L. Zhang, L. Zhang, D.Tao, "Stacked Convolutional Denoising Auto-Encoders for Feature Representation," IEEE Transactions on Cybernetics, vol. 47, pp. 1017-1027, 2017.

[8] B.Du, Z.Wang, L.Zhang, L.Zhang, W.Liu, J.Shen, D.Tao, "Exploring Representativeness and Informativeness for Active Learning," IEEE Transactions on Cybernetics, vol. 47, pp. 14-26, 2017.

[9] D. Tao, X. Li, X. Wu, S. J. Maybank, "Geometric Mean for Subspace Selection," in IEEE Transactions on Pattern Analysis and Machine Intelligence, vol. 31, no. 2, pp. 260-274, Feb. 2009.

[10] D. Tao, X. Li, X. Wu, S. J. Maybank, "General Tensor Discriminant Analysis and Gabor Features for Gait Recognition," IEEE Transactions on Pattern Analysis and Machine Intelligence, vol. 29, pp. 1700-1715, 2007.

[11] C. Peng, J. Cheng, Q. Cheng, “A supervised learning model for high-dimensional and large-scale data," ACM Transactions on Intelligent Systems and Technology (TIST), vol. 8, pp. 1 - 23, 2017.

[12] Q. Cheng, H. Zhou, J. Cheng, H. Li, "A Minimax Framework for Classification with Applications to Images and High Dimensional Data," IEEE Transactions on Pattern Analysis and Machine Intelligence, vol. 36, pp. 2117-2130, 2014.

[13] P.S. Hiremath, C.J. Prabhakar, "Face Recognition Using Symbolic KPCA Plus Symbolic LDA in the Framework of Symbolic Data Analysis: Symbolic Kernel Fisher Discriminant Method,” International Conference on Advanced Concepts for Intelligent Vision Systems, pp. 982-993, 2008.

[14] H. Roy, D. Bhattacharjee, "Heterogeneous face matching using geometric edge-texture feature (GETF) and multiple fuzzy-classifier system,” Applied Soft Computing, vol. 46, pp. 967 - 979, 2016.

[15] Q. Q. Tao, S. Zhan, X. H. Li, T. Kurihara, "Robust face detection using local CNN and SVM based on kernel combination," Neurocomputing, vol. 211, pp. 98-105, 2016.

[16] image dataset: https://github.com/StephenMilborrow/muct

[17] P. Dinesh Kumar, Dr. B. Rosiline Jeetha, "Unaffected Serial Prophecy based Filter Technique for Noise Removal in Facial Expression Recognition Images,” International Journal of Civil Engineering and Technology (IJCIET), vol. 8, pp. 497 - 506, 2017.

[18] P. Ekman, T. Huang, T. Sejnowski, J. Hager, "Final Report to NSF of the Planning Workshop on Facial Expression Understanding", 1992.

[19] EwaPiatkowska "Facial Expression Recognition System", Master's Thesis Technical Report. 